\title{
REVERSING THE ARROW OF EXPLANATION IN THE RELATIONAL BLOCKWORLD: WHY TEMPORAL BECOMING, THE DYNAMICAL BRAIN AND THE EXTERNAL WORLD ARE ALL "IN THE MIND"
}

\author{
MARK STUCKEY ${ }^{1}$ and MICHAEL SILBERSTEIN ${ }^{2,3}$ \\ Departments of Physics ${ }^{1}$ and Philosophy, ${ }^{2}$ Elizabethtown College \\ Elizabethtown, PA 17022, U.S.A. \\ (stuckeym@etown.edu, silbermd@etown.edu) \\ MICHAEL CIFONE \\ ${ }^{3}$ Department of Philosophy, University of Maryland \\ College Park, MD 20742, U.S.A. \\ (cifonemc@wam.umd.edu)
}

\begin{abstract}
We introduce the Relational Blockworld (RBW) as a paradigm for deflating the mysteries associated with quantum non-separability/non-locality and the measurement problem. We begin by describing how the relativity of simultaneity implies the blockworld, which has an explanatory potential subsuming both dynamical and relational explanations. It is then shown how the canonical commutation relations fundamental to non-relativistic quantum mechanics follow from the relativity of simultaneity. Therefore, quantum mechanics has at its disposal the full explanatory power of the blockworld. Quantum mechanics exploits this expanded explanatory capability since event distributions among detectors per the density matrix follow from spacetime relations (symmetry group) alone. Thus, the event distributions of non-relativistic quantum mechanics follow from a blockworld wherein spacetime relations are fundamental. Per RBW "quantum mysteries" are deflated and the implications for consciousness and the perception of temporal flow and absolute becoming are explored. We conclude that given RBW, consciousness is no less fundamental than any "physical" feature of the world such as brain states. Further, active consciousness is needed to explain the illusion that it is a dynamical world and consciousness in its most fundamental state is relational and non-local
\end{abstract}

Keywords: Quantum Non-locality - Relational Blockworld - Fortuitousness - Consciousness - Absolute Becoming

\section{Introduction}

The Relational Blockworld (RBW) can be understood in two ways: (1) as a framework providing computational and metaphysical structures which can resolve most (if not all) of the tensions between non-relativistic quantum mechanics (QM) and special relativity (SR) and (2) as providing an inter- 
pretation of QM that deflates its conceptually problematic features, such as entanglement, non-locality, and quantum measurement. As an item of empirical metaphysics, RBW describes a reality in which the essence of SR (the relativity of simultaneity) and the essence of QM (non-commutivity, non-locality and non-separability) are both true of that reality. As an item of empirical science, RBW provides the computational formalism to model phenomena non-dynamically in an irreducibly relational fashion. The goal of the RBW program is to unify QM and SR in an empirically verifiable, non-dynamical account of reality.

In order to accommodate their essential features, QM and SR cannot both be afforded fundamental status in a unifying theory. Namely, there cannot be both definite classical events constituting a Minkowski manifold and physical observables that are non-commutative, non-separable and nonlocally correlated. Per RBW, we can preserve the essence of both SR and QM if spacetime symmetries are considered fundamental, i.e., spatiotemporal relations are fundamental to relata. The conceptual price for this picture of reality - a radical spatiotemporal relationalism and a radically non-dynamical perspective - is dissonance with the way physics typically models reality. However, RBW is empirically compatible with both SR and QM, and affords a "middle way" out of the well-known conflict between them. In providing a spacetime basis for both SR and QM, RBW manages to capture the essential elements of our best spacetime theory and our best theory of matter, but gives up (i) the fundamentality of "trans-temporal objects," (ii) dynamical physical processes, (iii) the substance/property model of phenomena and (iv) time as a fundamental ingredient in physical explanations. Section 2 discusses in more detail how RBW resolves the tensions between QM and SR.

According to RBW, irreducible spatiotemporal relations in a blockworld explain physical phenomena (like the clicks in a measurement instrument, etc.), not transtemporal objects manifesting certain properties and interacting with other transtemporal objects. RBW explains by giving the geometric structure of spacetime symmetry relations - i.e., the global relational dependence of various regions of spacetime with each other. Geometry is fundamental in RBW; all other physical facts are manifestations, if you will, of the geometric structure of spacetime symmetries. RBW radically diverges from the way physics typically models reality, i.e., by presupposing trans-temporal objects, idealized as test particles with mass, under the governance of a class of dynamical laws and kinematical principles.

Consequently, the Hilbert space of QM, while computationally essen- 
tial, is replaced conceptually with geometry in RBW. Thus Hilbert-space quantum mechanics, by which we mean the non-commutative structure of observables, entanglement and non-locality represented in the Hilbert space formalism, is not fundamental. Rather the geometry, uniquely determined by the spacetime symmetries basic to RBW, is fundamental. Not only does RBW advocate the view that spacetime geometry of Minkowski spacetime can satisfactorily explain, and even deflate, the so-called conceptual "mysteries" of QM, but RBW explains how QM follows from the geometry of a suitably chosen spacetime symmetry structure [1]. According to RBW the Hilbert space representation of physical observables, and the whole paradigm of state-space physics, are merely calculational tools - they are not metaphysically fundamental. The focus of Section 3 is to show how RBW deflates the various well known quantum mysteries.

In short, the three main tenets of RBW are: (1) the relativity of simultaneity (RoS) implies a BW view of reality, (2) QM is a consequence of a spacetime structure which respects RoS, (3) QM is a manifestation of the irreducibly relational symmetries of spacetime, and is not a dynamical theory of matter in motion, i.e., nothing is "quantized;" there are no "particles," "waves," etc., moving/acting in spacetime according to some dynamical equation of motion, such as Schrödinger's equation. The majority of the paper, Section 4, is devoted to drawing out the consequences of RBW for the experience of time, change and the status of consciousness. We conclude that consciousness is as fundamental in RBW as anything else, and that like everything else in RBW, it is fundamentally relational, non-separable and non-local. We also show why fundamental consciousness must be invoked in RBW to explain the persistent illusion of a dynamical world or "moving now." It is the dynamics of consciousness that explains the experience/illusion of a dynamical world and not the other way round.

\section{RBW Resolves Tensions Between QM and SR}

\subsection{The relativity of simultaneity implies $B W$}

The property of simultaneity is defined by a spacetime foliation into spacelike hypersurfaces. A spacelike hypersurface is a plane of simultaneity, i.e., what we refer to as "space" or "the universe" at an instant of time. According to SR, a collection of observers A at rest with respect to one another will foliate spacetime differently than another such collection of observers B in motion with respect to A. Further, there is no reason to grant preferred status to either foliation, so SR implies the relativity of simultaneity. 
As a consequence of RoS, consider an observer Alice in A passing an observer Bob in B. Except for one another, Alice and Bob will disagree on who exists simultaneously with them at that instant of time (call it "today"); people at rest with respect to Alice will exist simultaneously with her "today," while those at rest with respect to Bob will exist simultaneously with him "today." According to SR, the people in Bob's plane of simultaneity will exist with people in Alice's past and future, and vice-versa. So, Bob and Alice exist together "today" and people in Bob's "today" exist together with people in Alice's "tomorrow" and "yesterday." Likewise, people in Alice's "today" exist together with people in Bob's "tomorrow" and "yesterday." If there is no empirical means of discrimination, then both Alice and Bob are justified in their designations of who exists with them "today," so their pasts and futures are as real as their presents. This interpretative consequence of the RoS is known as the blockworld.

Per the blockworld (BW), temporal as well as spatial location must be physically insignificant in some deep sense, and must be a matter of one's perspective rather than a property of the spacetime events themselves. The temporal and spatial locations of an event are unique to one's frame of reference in a more profound manner than implied by the intuitive Galilean coordinate transformations of Newtonian physics. As a consequence, conscious beings themselves, as observers in the spacetime of SR, also lack absolute spatiotemporal locations. Conscious observers do not carry an intrinsic "now" that is somehow mixed into the nature of their being to single out points along their worldlines in spacetime. If a "now" continually highlights different points along a particular worldline, then there exists movement with respect to another temporal dimension, i.e., some 'meta-time'. Meta-time invoked for the purpose of a moving "now" is empirically inconsequential otherwise, so in the interest of parsimony it is ignored by physics. Thus to imbue worldlines with "nowness," without invoking a superfluous meta-time, requires all points on all worldlines be equally endowed, thereby rendering the concept of "nowness" useless.

In summary, RoS says different space-like foliations of the spacetime manifold obtain and no space-like foliation is preferred, i.e., no observerindependent physical features of the spacetime manifold can be used to distinguish one foliation from the infinitely many other possible foliations. BW is the straightforward extrapolation of RoS to the co-reality of the past, future and present for all observers. Thus, the spirit of SR is the relativistic democracy afforded to all foliations of Minkowski spacetime and any theory purporting to be empirically equivalent to SR, while adding a 
preferred frame, violates this essential spirit and is rejected by BW. In this sense, BW accepts an unmodified SR, i.e., one true to the "principle" version of SR developed by Einstein, as opposed to the "constructive" version of SR Lorentz tried to develop before Einstein that presupposed a fixed background Newtonian spacetime along with an "aether" to explain the signature of the spacetime metric [2].

\section{2 $Q M$ resides in a $B W$}

Perhaps surprisingly, BW is germane to non-relativistic quantum mechanics. This follows from the work of Kaiser and Bohr \& Ulfbeck who showed the canonical commutation relations of $Q M$ follow from the relativity of simultaneity. Kaiser writes [3],

For had we begun with Newtonian spacetime, we would have the Galilean group instead of [the restricted Poincaré group]. Since Galilean boosts commute with spatial translations (time being absolute), the brackets between the corresponding generators vanish, hence no canonical commutation relations (CCR)! In the $[c \rightarrow \infty$ limit of the Poincaré algebra], the CCR are a remnant of relativistic invariance where, due to the nonabsolute nature of simultaneity, spatial translations do not commute with pure Lorentz transformations.

Bohr \& Ulfbeck [4] realized that the "Galilean transformation in the weakly relativistic regime" is needed to construct a position operator for QM, and this transformation "includes the departure from simultaneity, which is part of relativistic invariance." Regarding the commutator of the "weakly relativistic boost" with a spatial translation they note, "The product of the coordinate transformations [a 'weakly relativistic' boost and a spatial translation] taken in the opposite order are seen to differ by a time displacement..." It is precisely this "time displacement" which is responsible for RoS. They write [4],

"For ourselves, an important point that had for long been an obstacle, was the realization that the position of a particle, which is a basic element of nonrelativistic quantum mechanics, requires the link between space and time of relativistic invariance."

Thus, the essence of QM - its canonical commutation relations - is the result of RoS and QM may be regarded as residing in a BW akin to that of Minkowski spacetime. 


\subsection{From a classical to a relational blockworld}

QM predicts the existence of physical observables which are non-commutative, non-separable and which can manifest non-local correlations at arbitrary distances. QM manifestly contradicts our ordinary classical picture of reality, and as such isn't obviously compatible with what might be called the "classical" BW view provided supra. RoS is organic to QM as shown in the preceding section, but can BW, as an interpretation of RoS, accommodate QM?

Many philosophers, like Huw Price [5] and Gordon Fleming (with H. Bennett) [6], who are inclined to think about the theoretical problems between SR and QM, have proposed very novel theories which seemingly accommodate QM in a BW setting. Price takes issue with the temporal bias inherent in the common cause principle, which states that correlated experimental outcomes exist because of some common event in their past light cones and never the converse. Price proposes, rather, a time-symmetric QM in a BW setting. For Price, the arrow of time can point from past to future or future to past in order to explain the spacetime relationship between a common event in the past light cones of correlated experimental outcomes and those experimental outcomes. Unlike Price, Fleming advocates relativizing quantum mechanical properties like entanglement to hyperplanes of simultaneity in a Minkowski setting rather than trying to devise a timesymmetric interpretation of QM. Thus, at least in principle, Fleming's view preserves the spirit of BW by taking entanglement to be a relation between a physical system and some hyperplane and then being democratic about all the families of hyperplanes per RoS.

While perhaps only implicit in both Price and Fleming's proposals, spatiotemporal relationalism is made explicit in RBW wherein the salient geometric structure of BW is used to explain, rather than merely 'house', QM. By doing so, RBW makes full use of the expanded explanatory potential of BW that is obtained via relationalism, and dynamical contrivances aren't required to accommodate $\mathrm{QM}$ phenomena. In fact, according to the relationalism of RBW, spatiotemporal relations exist independently of, and fundamental to, relata. This radical relationalism avoids the necessity of invoking classical, pseudo-classical or even quantum mechanically "entangled" entities with their own properties, changing over time, to explain fundamental physical processes. And, while other proposals merely render QM and SR compatible, RBW unifies them in that both emerge from the same basic geometric structure. In these respects, $\mathrm{RBW}$ is quite distinct from proposals common in the literature today that try to square QM and 
SR in a BW setting.

It is this relationalism which distinguishes RBW from BW in a classical setting such as SR, and allows for the explanation of quantum phenomena. In short, a classical BW becomes radically relational to accommodate certain features of QM. But this move is not made merely to accommodate quantum phenomena in a relativistic setting - we have quantum field theory for that. Rather, this move is made to deflate the "mysteries" of non-relativistic quantum mechanics while maintaining harmony with SR. And, the use of spatiotemporal relationalism is entirely justified by the formalism of QM.

That is, Bohr, Mottelson \& Ulfbeck [7] have shown the density matrix can be obtained via the spatio-temporal symmetries of the experimental configuration (symmetry group) rendering concepts such as the Hamiltonian, mass and Planck's constant ancillary. This implies that detector clicks are not caused by impinging particles or collapsing waves that move through or occupy the space between the source(s) and detector(s). Bohr, Mottelson \& Ulfbeck write [7], "Indeed, atoms and particles as things are phantasms (things imagined)." Bohr \& Ulfbeck [8] call this the Theory of Genuine Fortuitousness and write [4],

"It would appear, however, that the role of symmetry in relation to quantal physics has, so to speak, been turned upside down, and it is the purpose of the present article to show that quantal physics itself emerges when the coordinate transformations (the elements of spacetime symmetry) are recognized as the basic variables."

Now according to BW, as explained supra, RoS implies all spacetime events are equally real, whether they be labeled past, present or future relative to some observer. The reality of all events is "hidden" from beings "inside" the block, because they are relegated to a single perspective. The sense in which the totality of facts is hidden from the view of any one perspective in the BW gives rise to a "need," so to speak, for constructing dynamical explanations of, say, quantum phenomena. But in the block, "change" or "time" isn't a fundamental ingredient in the ultimate explanation of reality since nothing changes in a BW. By building upon BW, RBW employs a non-dynamical perspective, and dynamical explanations such as "genuine fortuitousness" are not necessary at the fundamental level of reality.

With this result, we have the physical motivation to reject the view 
that there are objects with intrinsic properties, or that there need be relata standing in the relations at the fundamental level of reality when explaining physical phenomena. Irreducible symmetry relations are all that exist at the fundamental level of reality. Consequently, RBW is radically relational and non-dynamical.

In conclusion, we understand that both the facts of SR and the facts of QM are facts about the same thing - spatiotemporal symmetries understood to be irreducible relations in a BW. This motivates RBW and reveals a deep unity between SR and QM. Consequently, in RBW we do not find a fundamental tension between SR and QM.

\section{RBW Deflates "Quantum Mysteries"}

$\mathrm{RBW}$ is a blockworld in which spatiotemporal relations are fundamental. A blockworld is a spacetime in which the future, past and present are equally real. Thus, presentism does not obtain in a BW and there is no uniquely "evolving universe" or "unfolding now." Every event that will happen or has happened just 'is' in a BW. In this sense, nothing about a BW can change, so the collapse (qua dynamical process) of the wave function must be a fundamentally epistemological fact about the state-space formalism that does not directly capture the ontological facts of a relational blockworld. That is, the wave-function qua state-space representation of QM is a calculational device, whereas the relational spacetime symmetries of an experimental arrangement, that give rise to quantum statistics, is the deeper ontological story of QM. Geometry is fundamental to Hilbert space, to use a slogan. Thus, BW eliminates the measurement problem trivially.

Quantum non-locality and non-separability are likewise handled trivially since RBW assumes spatiotemporal relations are fundamental in a BW. Correlations between space-like separated events that violate Bell's inequalities are of no concern as long as spatiotemporal relations in the experimental apparatus warrant the correlations. There is no need to satisfy the common cause principle, since non-local correlations are not about "particles" impinging on measuring devices. Rather, the non-local correlations derive from the spatiotemporal relations in the construct of the experiment. There are no influences, causal mechanisms, etc., because non-locality is a relational property that is precisely described by the spatial translations, rotations and reflections of any given experimental arrangement. Nothing happens in a relational blockworld, so there is nothing for such inherently dynamical processes and entities to do. One can invoke dynamical stories to account for quantum non-locality, but these stories will invariably be 
"error theories" in the sense that these dynamical stories do not express the deeper facts fundamental to a relational blockworld.

The conceptual trouble with quantum non-locality, quantum non-separability or entanglement is a consequence of our seemingly dynamical perspective 'within' a relational blockworld. The trouble is with us, not the world, so to speak. In trying to explain the spatiotemporal distribution of detector clicks as caused by or as determined by impinging particles (carrying with them their own properties), the standard account of QM in a spacetime setting assumes a Galilean background spacetime in which quantum states evolve. Since simultaneity is (in principle) absolute in Galilean spacetime, dynamical accounts of QM seem natural. If one is worried, though, about the tensions between QM and SR, then not all dynamical explanations will be so "natural." If SR is true and reality is like a blockworld, then quantum non-locality, non-separability, etc. better not violate RoS. Thus, nothing we say about quantum non-locality ought to allow us to send superluminal signals, for example. As well, if reality is like a BW, then nothing happens in the sense that nothing new "comes into being." Preand post-measurement facts are "already there" and don't come to be after a measurement "interaction." There is nothing ontologically unique about measurement events - they don't "change" anything. We can't think that a particle, in interaction with a measurement device, brings some fact into existence that wasn't already true. Particles don't "go" anywhere and don't "interact" with anything. Nothing "happens:" the past, present and future are equally real. Non-dynamical explanations must at least respect the reality of all spatiotemporal events.

$\mathrm{RBW}$ goes beyond mere non-dynamism when it comes to explaining quantum phenomena: QM is a manifestation of the geometry of spacetime symmetries where simultaneity is relative. Thus, RBW does not assume that QM is independent of Minkowski spacetime - rather QM is derivative from the (reduced) spacetime symmetries of Minkowski spacetime itself. Quantum facts are facts about the irreducible spatiotemporal relations of a given physical system, not facts about the behavior of particles, or the interactions of measurement devices and wave-functions, etc. There is no "collapse of the wave function" because there are no real quantum states just "states" (if you will) of the entire spacetime configuration of a physical system fixed at once. Dynamical talk is simply a crude way of trying to describe global, static, spatiotemporal dependency relations between various regions of spacetime in a given experimental situation.

According to RBW, reality is fundamentally relational and non-dynam- 
ical, but representable dynamically. From the point of view of RBW, then, novel quantum phenomena such as non-separability are conceptually problematic only in the attempt to formulate a dynamical explanation for something that is irreducibly relational and non-dynamical. In fact, all phenomena are "non-separable" via the spatiotemporal holism of RBW. All of which has profound implications for the way we think about phenomenal consciousness and the way we explain our experiences of absolute change and temporal becoming.

\section{Implications of RBW for the Experience of Time, Change and the Status of Consciousness}

Given that in RBW temporal flow and absolute becoming are not external features of the world in their own right, there is no special "Now" moving from past to present, time does not flow or "go by," etc., then we must explain the experience of these things without recourse to these things as actual features of the world. That is, we must provide an explanation for the experience of absolute becoming and temporal flow in a relational blockworld in which presentism is false. What follows is just a short list of the phenomenological features of temporal experience (the various "psychological arrows of time") that must be explained in order to explain our experience of time and change (see Dainton [9] for a more detailed discussion):

1) Why, at any given moment, is our conscious awareness confined to such a small part of spacetime that we dub "the now?"

2) What gives some experiences, by definition those that feel real to us at any given moment, their feeling of presentness? Why do we experience some pains as memories and others as having the property of "nowness" or presentness?

3) Why are feelings of presentness and "realness" so inextricably connected? What explains the feeling that to be real is to be present, after all even memories only feel real when dragged into the present and conscious attention is focused upon them. It seems to be a transcendental truth that what separates real experience from, say, mere memories is "being appeared to presently."

4) What explains the experience that things change and that the experienced present is continually advancing into the future? Why do we feel that our lives unfold in the direction of the future-that time has a direction? For example, we can always tell whether a film is being run forward or backward, why is that? If time started going backwards globally, after a 
while, no doubt we would again feel that our lives were unfolding into the future.

5) The experience of the "phenomenal flow" of time, we experience time as passing even when there are no detectable changes in our environment.

6) The temporal coherence of our stream of consciousness or succession of experiences such that each phase seems "phenomenally bonded" to its immediate predecessor and successor.

7) Why do we have detailed knowledge of (or greater epistemic access to) the "past" but not the "future"?

8) Why do we have such an asymmetry regarding attitudes, feelings, explanations and decision making about the "future" if "past" events are equally real? Why, for example, do I fear future pains but not past ones? Why do we generally explain later events (such as dying) with reference to earlier events (such as getting shot) and not the other way around?

9) What explains the phenomenology of the "specious present" — that experience comes in packets of meaningful duration (think of a melody or reading passages) say as opposed to durationless instants or spacetime points?

There are only so many logical possibilities open to us when it comes to explaining (or not) our experience of time and change:

1) Various asymmetries or features of internal mental (or neural) processes.

2) Various asymmetries or features of the external world.

3) Various asymmetries or features of both internal mental processes and the external world.

4) Neither internal mental processes nor the external world has the resources to explain our experience.

Of course there is no reason to believe that all of the preceding psychological arrows of time will be explained in exactly the same way. For example, the experienced asymmetry between past and future or the experience of change might be explained by asymmetries in the external world such as the thermodynamic arrow of time plus memory and the experience that irrespective of change time is always "flowing" might be explained by other mechanisms either in the world and/or in the brain.

That said there are a couple of misnomers here that need to be dispelled in order to make progress. First, that if the blockworld is true and contrary to presentism we do not directly apprehend the "moving now" or global metaphysical present, then we can only appeal to (1) above when it comes to explaining our experience of time and change, and thus explaining 
the experience of temporal flow and absolute change is much harder given blockworld. Second, that if presentism is true we can appeal to both mental processes and real asymmetries in the external world and therefore our experience of temporal flow and absolute becoming are no great mystery.

These claims are misnomers because (i) many external asymmetries (such as causal patterns) still exist even in a blockworld and can be called on by all to help explain experience, (ii) it is not clear how we would perceive a "moving now" even if it did exist and (iii) explaining the various psychological arrows of time turns out to be quite hard regardless of whether blockworld or presentism is true. As for (i) the following is a nonexhaustive list of external asymmetries that anyone, regardless of whether blockworld or presentism, is true, can appeal to in order to try and explain our experience of time and change (see Dainton [9] for a more detailed discussion):

1) Entropic asymmetry: the second law of thermodynamics states that entropy increases over time.

2) Causal asymmetry: some events are regarded as causes of other events and causes generally precede their effects.

3) Fork asymmetry: we often find later events that are correlated or caused by a single earlier event, for example everyone exposed to radiation gets sick at a later time. The inverse fork is much rarer.

As for (ii) while our perceptions appear to immediately inform us about the order and duration of events, we cannot apply any of the usual models of perception to explain this fact. The problem is that order, duration and other temporal "features" unlike, say, shape and color, are not "objects" or "properties" of perceptual states in any standard sense. To perceive the order and duration of events we have to be aware of the events themselves, whereas with color and shape we can focus on those features to the exclusion of other features of an event or entity. The point is that order, duration, the "moving now," etc., do not appear to be external features of events in the way that shape and color are, and therefore it is hard to explain our experience of them by appeal to the causal theory of perceptual knowledge as we do with say color experiences. Whereas we have identified areas of the brain responsible for perceiving and representing color and shape related physical phenomena, it is not clear that there is any part of the brain devoted to perceiving and representing an externally given "moving now." This leads some people to believe that the experience of the "moving now" is a projection of mind or brain rather than a perception, perhaps 
even a transcendental ground of experience as Kant would have it.

The point of (iii) is that regardless of blockworld or presentism, it is not yet clear to us what external and/or internal features account for our experience of temporal flow and absolute becoming. This much is clear, both blockworld and presentism can (and often do) appeal to many of the same external and internal features in order to explain our experience of time and change, and neither view has produced an answer leading to a scientific consensus - it is still a mystery. While blockworld, unlike presentism, cannot appeal to a special "moving now," the truth of presentism, etc., in order to explain the various psychological arrows of time, it is far from clear what extra explanatory mileage presentism gains from such an appeal. And again, the blockworld can also appeal to such external asymmetries as the causal (light-cone) structure given by Minkowski spacetime, the "radiative" asymmetry, the entropic asymmetry, etc. Blockworld can acknowledge that these and other external features of the world often contribute to the illusion of a "moving now" and absolute becoming. But as we shall see, such external features of the world are at best necessary conditions for our experience of time and change.

Indeed, just as there is an "explanatory gap" (and perhaps an ontological one) between brain processes and "phenomenal consciousness," so there is an explanatory gap between on the one hand time and change as conceived in the physics of blockworld and on the other the experience of temporal flow and absolute becoming. In fact the problem of explaining our conscious experience of time and change is in part a subset of the "hard problem of consciousness." Some might even argue that the experience of "nowness" slipping into the future is the very essence of phenomenal consciousness. And as we shall see, the hard problem becomes much harder in a blockworld and it takes on a certain ineluctable shape.

However, that said, even in a world dynamically conceived such as presentism there is a huge disconnect between the "flow" of physical processes (the external "arrows of time") and the subjective experience of temporal becoming. That is, there is no obvious isomorphism between physical processes and temporal experience (though some neuroscientists may hope to find some in the brain). In addition to showing us the disconnect on the one hand between time and change as experienced and the various external or physical arrows of time on the other, the following well-known examples also illustrate the necessity of normal brain function in "producing" our ordinary experience of temporal flow and absolute becoming:

The 0.5 second time lag in perception, the experienced present is actu- 
ally of the recent past as there is a typical time-lag between initial stimulation and experience of 0.5 seconds. Obviously we are not aware of this time-lag. The more complicated an incoming stimulus, the longer it takes the brain to process.

Coherent and complex perceptual experiences involving multiple sensory modalities are formed from sensory stimuli that travel at different speeds, such as the speed of light, sound, smell, etc. Therefore each kind of sensory data that will ultimately form a particular coherent experience for the observer arrives at the brain for processing at different times. Furthermore, all of these different sensory signals are processed in different parts of the brain. The visual stimuli alone such as that pertaining to motion, color, distance, size, and "higher-level" processes such as facial recognition all get processed in different parts of the brain. But we do not, for example, experience the visual elements of an event before the auditory features even though light travels faster than sound. So how is it that people (with normal brains at least) experience perfectly synchronized and coherent events or conscious perceptions? The complete answer to this "binding problem" is unknown, but we do know that it involves the brain constantly "backdating," rearranging or reversing conscious perception in time. The brain backdates conscious perceptions to the time when the stimulus first entered the brain. Consciousness of events are backdated so that awareness seems to arise at the same time as the events actually take place. Part of this is explained by different processing times in the brain, for example, auditory signals are processed more quickly than visual ones.

However a differential in processing times for different stimuli is not the whole story. For example, Libet [10] discovered that if we directly stimulate the appropriate spot on the somatosensory cortex that is connected to the hand and then 150 milliseconds later we stimulate the hand itself, the subject reports the hand stimulus as coming first. This is very puzzling because the "real external" time to process both signals is the same. Somehow the brain flipped the order of events in conscious experience.

Another good example of the constructed nature of time perception is the brain mechanism of saccadic suppression. Why, given how rapid eye movements are, do we not feel motion induced nausea and experience the world as blurred? Part of the answer is saccadic suppression. This is a brain mechanism that interferes with vision during eye movements and blinks. Vision is at least in part shut down when your eye is in transition and thus we suffer neither nausea nor jerky images. The amount of time during the day in which vision is shut down is not trivial, "adding up all the little 
snippets of the running movie that constitutes daily life that are 'lost' due to saccadic and blink suppression amounts to a staggering 60 to 90 minutes each day," Koch [11]. Why then do we not experience blank periods in our visual awareness everyday? Most neuroscientists believe that there must be some "trans-saccadic integration" brain mechanism that "fills in these intervals with a 'fictive' movie, a composite of the image just before and just after the saccade," Koch [11].

If perception of temporal flow, the order of events, duration and other time-related features of experience are constructed and active projections of cognition, then intersubjective agreement under normal conditions plus individual local "nows" might help create the illusion of a shared "moving now" and absolute becoming "out there" in the external world. Of course there are many external contingencies that contribute to this intersubjective agreement such as the fact that the time scale of perception is short compared to the time scales upon which key features of the macroscopic environment change or vary, individuals are moving relative to one another at velocities small compared to $c$, the light travel time between individuals in an inertial frame in which they are nearly at rest is small compared to the time scales of perception, etc.

However normal internal or cognitive conditions are also necessary for ordinary time perception and they do not always obtain. We now have good evidence that there are one or more "clocks" in the brain. One such brain clock is a loop of dopamine-generated neural activity which flows between the substantia nigra in the base of the brain (where dopamine is produced), the basal ganglia, and the prefrontal cortex. Each "tick" of this clock is the same time it takes for the nerve signals to complete the loop. All neural events that occur within that time are experienced as a single moment. The average tick is about one-tenth of a second, but they do vary considerably. Many external events may occur within a particular cycle or tick of the brain clock, such as the flapping of an insect's wings, but they will be perceived as one event. If two flashes of light are presented to someone with less than a one-tenth of a second gap between them, they will be perceived as one flash only. If the two flashes are far enough apart so as to be in separate cycles or ticks of the brain clock, they will be perceived as such.

As a result of this data and more, some neuroscientists such as Koch [11] now believe that all perception, rather than being continuous, is actually discrete: "perception might well take place in discrete processing epochs, perceptual moments, frames, or snapshots. Your subjective life could be a 
ceaseless sequence of such frames." The suggestion here is that relative to each such snapshot the perception of sensory qualities such as color, depth and even motion would be constant. On this view motion is experienced not because of a change in position between two consecutive snapshots such as happens with film, but because motion is represented within a single snapshot by somehow being suggestive of movement. Imagine for example a still photograph of someone in a running posture. It is hypothesized that if conscious perception does take place in discrete moments that the perception of the passage of time might in part be a function of the rate at which snapshots occur. For example, the kind of "protracted duration" or slowing down of the passage of time experienced during accidents might result from more snapshots occurring per unit time and thus the same one-second interval will now be divided into more snapshots than normal resulting in the feeling that time is passing much more slowly. There is a rare alteration in time perception that sometimes happens to people with severe visual migraine dubbed "cinematographic vision" by Oliver Sacks (Koch [11]). When this occurs, time is experienced as discontinuous like a succession of stills with nothing in between in which the illusion of motion has been lost. Koch [11] hypothesizes that in such cases "the migraine may have temporarily inactivated the cortical motion areas," thus providing some first-person evidentiary support for the discrete theory of perception.

There are several well-known examples in which, due to brain damage, illness, trauma, shock, etc., the timing mechanisms in our brain are disrupted and produce radically altered states of consciousness. People with Parkinson's disease, in which the neurotransmitter dopamine is depleted, experience the passage of time differently. If you ask the average person to say "now" after they think a minute has passed, they will do so after about 40 seconds. The person with Parkinson's however will on average say "now" after about 60 seconds has passed. In one well-known case a 66-year old man found that he could not drive or watch TV because the cars and TV images seemed to be on "fast forward" and were zooming by him. When the "minute-test" was applied to him, he didn't say "now" until five minutes had passed. It was later discovered that the man had a growth in his prefrontal cortex. Catatonic patients who recover sometimes report their experience while catatonic as being like "stuck on pause," their memories of that time period suggest that time did not pass for them and there was no change in conscious states, though they were aware. We have all experienced alterations in our experience of temporal flow and duration during fever, accidents, etc. 
The normal function of memory and its relationship to perception is also an important condition for our experience of the passage of time. One major reason we "sense" time passing is that our perceptions and memories combine in a particular sort of way over our entire life. At each moment of conscious awareness I have memories of previous moments from a few seconds back to many years back. This contributes significantly to the illusion that time passes, that absolute change occurs and it helps explain why I have the belief that time is really dynamically unfolding. When this memory mechanism is disrupted it causes extreme alterations in perceptions of time. For example, patients with Korsakoff syndrome cannot make new long term memories beyond those they had at the time of their brain damage and whatever enters their short term memory is forgotten in a couple of minutes or less. Such people do not experience time as passing as we do and in some extreme cases they do not believe they are aging until forced to view themselves in a mirror; they are horrified of course, but not for very long as they cannot retain the memory of what they saw. One very extreme example of amnesia is the case of Clive Wearing, "a gifted musician and scholar, he suffered a viral brain infection that almost killed him and destroyed parts of both temporal lobes. Clive consciously experiences only the present. He has no childhood, no past," (Koch [11]). Having different memories at different stages in our lives helps explain the illusion that times passes. The right kind of patterns of memory accumulation is an important part of the story about our sense that time passes.

Regardless of whether asymmetries of both internal mental processes and the external world are necessary to explain the illusion that time passes, there is no denying that our experience of the world and our introspective processes reveal a universe that has very ordered and unique patterns of organization that bespeak of unfolding dynamical processes along the temporal axis. After all, there would be no past, present and future at all if spacetime did not have timelike directions. There would be no approximate intersubjective "now" if we did not exist on timelike worldlines, but rather existed on spacelike worldlines. Of course the internal and external asymmetries are not completely orthogonal. No doubt there are features of the very same laws of physics that give rise to the external asymmetries that are also necessary for the very existence and functioning of the brain-mind.

The point is this, the distribution of events as experienced by us over the course of our lives and the various asymmetrical patterns in the world as seen from our perspective could all exist even in a blockworld. We have seen that even holding all external features of the world constant, people's 
temporal experience can be radically altered. We have seen that our experience of temporal flow and change is largely a cognitive construction, all of which raises doubts that anything like real dynamical processes in the external world are either necessary or sufficient to explain our experiences. We have every reason to doubt that our experiences of time's passage and change are best explained by an objective global metaphysical property such as a "moving now."

So far so good, most of this is well-know to advocates of blockworld and most, therefore, are not too concerned by the argument from temporal experience against the static worldview. However there is a rub that no "blockworlder" that we are aware of has ever considered. We have learned that in order to explain our experience of time and change we are going to have to, at least in part, appeal to conscious brain processes whether it is a blockworld or not. Natural science such as cognitive neuroscience wants to explain the very existence of phenomenal conscious experience (such as the experience of a special "moving now"), by appealing to $d y$ namical brain processes. This is evident just from the brain mechanisms alluded to in this paper, such as the mechanism of "temporal binding" that makes coherent experience possible, the brain's clock that produces the discrete frames of perception, the "processing time" of all sensory modalities, the saccadic suppression mechanism, etc. All these mechanisms and all the models of brain mechanisms in general are essentially dynamical in nature. In neuroscience, in every case such as cognition, perception, memory, etc., the attempted explanation of these functions appeals to the dynamics of cell assemblies, neuronal firing rates, etc. Explanation of specific conscious states and specific cognitive functions in cognitive neuroscience is always in terms of "underlying causal mechanisms" in the brain or the "neural correlate of consciousness or cognition," both of which are inherently diachronic and dynamic conceptions of explanation. Thus, the fundamental working assumption of cognitive neuroscience and much of philosophy of mind is that matter in general and brain processes in particular are more fundamental than consciousness, both ontologically and explanatorily.

So what's the rub? In the blockworld there is no absolute motion or change, no dynamical processes actually exist. The block universe 'is', 'was' and 'always will be' as it 'is'. As Dainton [9] puts it: "Imagine that I am a God-like being who had decided to design and then create a logically consistent... block universe. Since the universe will be of the block-variety I will have to create it as a whole: the 'beginning', 'middle' and 'end' will come into being together. Well, assuming that our universe is a static block, even 
if it never 'came into being', it nonetheless exists (timelessly) as a coherent whole, containing a globally consistent spread of events." In a blockworld in which all events are equally real, any explanations proffered for any event (including those pertaining to conscious brain processes) that appeal to causal mechanisms/processes, non-linear dynamics, or more generally "becoming," "change," etc., must be error theories or merely compatibilist accounts of such processes. Certainly, as blockworlders have done since the beginning, we can cook up compatibilist versions of change, causation, becoming, etc., either by relativizing such notions to a frame of reference or by picking out certain invariant features in Minkowski spacetime, such as the light-cone structure, upon which to define these notions. But none of this changes the fact that all events in a blockworld are equally real and thus any talk about change, causation, becoming, etc., must be purely perspectival. All of which implies that brain "processes" do not literally cause (as in bring about or give rise to something that did not exist before) conscious "processes" and conscious processes are every bit as fundamental as brain processes. There is no absolute sense in which the better part of the universe's history unfolded without phenomenal consciousness and then conscious processes sprang into being and then became more sophisticated over time as the result of dynamically evolving brain processes.

Thus we find ourselves in the following dilemma: either explain phenomenal consciousness (or the illusion of such) by appealing to dynamical brain processes as we do in cognitive neuroscience, or explain the illusion of a dynamical world that appears to have temporal flow, change and becoming by appealing to the machinations of phenomenal conscious processes. At least when it comes to deciding which kind of explanation is more fundamental, we must choose one or the other option above - we cannot have both. Given blockworld, there is no absolute change, becoming, causation, etc, and therefore we must appeal to conscious experience (at least in part) to explain this illusion. And, given blockworld, we cannot a la cognitive neuroscience discharge consciousness as less fundamental or less explanatory than brain processes themselves. None of this suggests Cartesian dualism, but it does tell against physicalism as realistically conceived and it does speak for a kind of nondual or dual-aspect account of the relationship between mind and matter, as they are but two aspects or modes of a nondual blockworld. It is clear from all this that even in a classical blockworld (as opposed to RBW) there is an obvious sense in which the blockworld qua blockworld is a fundamental singular entity rather than something that can be decomposed or partitioned into discrete and autonomous parts in any 
absolute sense.

Anybody with standard dynamical physicalist intuitions about phenomenal consciousness should be asking themselves this question: forget about explaining the character of our experience such as temporal becoming and change, how can there be "beings" with conscious experiences at all in a static blockworld if nothing happens and all events and their various features (such as conscious states) are equally real? How can we explain phenomenal consciousness in a world in which evolutionary theory, molecular biology, cognitive neuroscience, etc., are merely heuristic devices in a static world? Assuming that conscious states are no exception to the block-nature of the world, what can a blockworlder say except "that's just the way this blockworld is, it's a brute fact that conscious beings exist and that particular conscious states bear whatever static correlations they do with particular brain states." If the physicalist finds this answer profoundly disturbing, then they have an internal conflict between our best physics and what they take to be our best explanations of consciousness and cognition from cognitive neuroscience.

There is one well-known physicist who tacitly at least acknowledges the inescapable brute nature of conscious states and their correlations with brain states in a static world, but nonetheless still tries to tell a story whereby brain states somehow determine, explain or are identical to conscious states. We have in mind Julian Barbour [12] who advocates what is arguably an even more radically static conception of the universe than our own RBW (see Barbour [12] for details). Barbour's interpretation of the Wheeler-Dewitt equation is that the universe is an $N$-dimensional configuration space wherein each point is a static three-space with one of infinitely many possible static configurations of matter-energy embedded in it. There is no temporal axis in Barbour's world. Barbour calls each of these points in configuration space a "Now." Each "Now" will of course have its conscious observers such that "any human experience is determined by that human's neurological state at a particular Now. A person will have different experiences at different Nows. Some of these will include representations of others, integrated in such a way as to be experienced as having happened earlier. Others will be integrated in such a way as to be experienced as perceived motion," (Healey [13]). In spite of his radically timeless universe, Barbour [12] says things that make him sound like a crude kind of mind/brain identity theorist when explaining, for example, the experience or illusion of motion: 
Could all motion be a similar deception? Suppose we could freeze the atoms in our brains at some instant. We might be watching gymnastics. What would brain specialists find in the frozen pattern of the atoms? They will surely find that the pattern encodes the positions of the gymnasts at that instant. But it may also encode the positions of gymnasts at preceding instants... The brain in any instant always contains, as it were, several stills of a movie. They correspond to different positions of objects we think we see moving. The idea is that it is this collection of 'stills', all present in any one instant, that stands in psychophysical parallel with the motion we actually see. The brain 'plays the movie for us', rather as an orchestra plays the notes on the score... If we could preserve one of these brain patterns in aspic, it would be perpetually conscious of seeing the gymnasts in motion.

Barbour must realize of course that given his claim that "Nows" are fundamental elements of reality, then brain states are no more fundamental than or explanatory than conscious states. Brain states do not exist prior in time to conscious states, nor do they "give rise to them" or cause them. Barbour's insinuation that brain states explain by merely "coding for" or being "isomorphic to" the conscious state they correlate with would be considered crude neuroscience even in a dynamical world. But the claim is even less well-motivated in his timeless world in which there is at best a brute correlation between a particular conscious state and a particular brain state; there is really nothing more to say about such correlations in such a world. In his more cautious moments Barbour [12] appreciates that at best his view can support a kind of naturalized psychophysical parallelism:

Nothing in the material world gives us any clue as to how parts of it (our brains) become conscious. However, there is increasing evidence that certain mental states and activities are correlated with certain physical states in different specific regions of the brain. This makes it natural to assume, as was done long ago, that there is psychophysical parallelism: conscious states somehow reflect physical states in the brain. Put in its crudest form, a brain scientist who knew the state of our brain would know our conscious state at that instant. The brain state allows us to reconstruct the conscious state, just as musical notes on paper can be transformed by an orchestra into music we can hear. 
However, even in the preceding passage embracing psychophysical parallelism, Barbour cannot resist making the additional claim that particular brain states code for particular conscious states such that in principle a super-duper neuroscience could read off the latter from the former. But again, in Barbour's timeless world the correlation or parallelism between particular brain states and particular conscious states is just a static brute fact, neither kind of state "explains" the other in any way. If neuroscience really could read off conscious states from brain states in Barbour's timeless world, that would just mean that we discovered that, in a purely a posteriori fashion, it just so happens that certain conscious states are always correlated with certain brain states, say across all "Nows."

The point of all this, and what Barbour does not fully appreciate, is that whether it be his timeless universe or the blockworld, the best one can say about the relationship between a particular conscious state and a particular brain is that they are correlated or if you prefer, "parallel" to one another. And of course there is no reason to believe that the correlations between conscious states and brain states are one-to-one. But, at least in Barbour's timeless world or the classical blockworld, the neuroscientist can take comfort in the fact that these static correlations exist between an individual's particular conscious states and particular brain states. This means that discovering the various static neural correlates of any given conscious state is as far as neuroscience can go in explaining such states in a blockworld.

Everything we have said so far about consciousness and the mind/body problem applies to a classical blockworld, but you will recall that we are defending a relational blockworld. In the relational blockworld things are even worse for physicalism and its standard dynamical conception of explanation regarding the mind and brain. As we have seen, in a classical blockworld it is still true that an individual's conscious states though not "determined by" their brain states, are at least correlated with those brain states. In the classical blockworld an individual's conscious brain states constitute a unified trans-temporal object that is local and separable with respect to the rest of the world. Even though a classical blockworld must be taken as a whole that is "open" to many different foliations, it is nonetheless also decomposable into individual events including conscious brain events. But recall that in RBW there are no fundamental events, trans-temporal objects, things, etc. In RBW all phenomena are non-separable and nonlocal via the spatiotemporal holism of RBW, and that includes conscious brain processes. Unlike classical blockworld, in RBW one cannot even ap- 
proximate the idea of the neural correlates of consciousness because such an explanatory schema presupposes that an individual's conscious brain states are separable and local with respect to the rest of the world, both of which will fail to obtain in RBW. In RBW not only do an individual's conscious states not "supervene" upon (are not determined by) their brain states, but they are irreducibly relational, non-local and non-separable in nature. In RBW brains are, after all, nothing but "emergent" phenomena from spacetime symmetries, they do not constitute autonomous systems anymore than measuring devices do in QM experimental set-ups. One must remember that RBW itself is not composed of anything, it is the one and only fundamental "entity" that there is, and it is not a thing, process or system.

All of this suggests that consciousness, like everything else in RBW, must also be irreducibly relational, non-local and non-separable. However there is no reason to believe that fundamental spacetime symmetries can account for conscious experience. This leads us to hypothesize that consciousness ("pure being"), which we believe in its most fundamental representation is responsible for, or identical with, the feeling of "nowness" or "presentness" that gives such experiences the "stamp of reality," should be modeled or represented via some other symmetry group. Like fundamental spacetime symmetries, fundamental "consciousness symmetries" are relational and non-local, and thus have no counterpart in the brain. Fundamental consciousness symmetries are no more a thing, object or process than spacetime symmetries are. Pursuing this analogy perhaps we could say that fundamental consciousness symmetries underlie individual conscious perspectives, perceptions, memories, and other such denumerable phenomenal states, in the same way that spacetime symmetries underlie the world of "dynamical" processes, physical events, and trans-temporal objects such as measuring devices, detectors and individual brain states.

In RBW it is the relational blockworld (spacetime symmetries), plus pure being (consciousness symmetries), plus some initial and boundary condition (a frame of reference or experimental configuration for example) that makes for individual experiential perspectives. Compare this with physicalism wherein the appropriate intrinsic, local and separable brain states, plus the appropriate distinct and local environmental context explain the existence of conscious "beings." In RBW, to be an individual is to be or occupy a conscious perspective in the relational blockworld. Individual existence is nothing more than the illusion of separateness. The relational blockworld is a seamless, nondual whole, individual existence is just the partitioning 
of the whole by the active mind. Thus, temporal becoming, the dynamical brain and the external world are all "in the Mind."

From the highest point of view the world has no cause. Once you create for yourself a world in time and space, governed by causality, you are bound to search for and find causes for everything. You put the question and impose an answer. Each moment contains the whole of the past and creates the whole of the future. In reality all is here and now and all is one. Multiplicity and diversity are in the mind. Everything is caused by all and affects all. The diversity is in you only. See yourself as you are and you will see the world as it is - a single block of reality, indivisible, indescribable. Your own creative power projects upon it a picture and all your questions refer to the picture. (Sri Nisargadatta Maharaj [14])

\section{References}

1. For this we rely on the work of Kaiser and Bohr, Ulfbeck \& Mottelson as described and referenced in what follows.

2. See Lorentz's "Electromagnetic phenomena in a system moving with any velocity less than that of light" (1904) for example, reprinted in The Principle of Relativity, Dover, New York.

3. Kaiser, G. (1981) Phase-space approach to relativistic quantum mechanics. III. Quantization, relativity, localization and gauge freedom, J. Math. Phys. 22, 705-714.

4. Bohr, A., and Ulfbeck, O. (1995) Primary manifestation of symmetry. Origin of quantal indeterminacy, Rev. Mod. Phys. 67, 1-35.

5. Price, H. (1999) Time's Arrow and Archimedes' Point: New Directions for the Physics of Time, Oxford University Press, Oxford.

6. Fleming, G., and Bennett, H. (1989) Hyperplane dependence in relativistic quantum mechanics, Found. Phys. 19, 231-267.

7. Bohr, A., Mottelson, B.R., and Ulfbeck, O. (2004) The principle underlying quantum mechanics, Found. Phys. 34, 405-417.

8. Personal correspondence to W. M. Stuckey dated 22 Dec 2004.

9. Dainton, B. (2001) Time and Space, McGill-Queen's UP, Montreal.

10. Libet, B. (2004) Mind Time: the Temporal Factor in Consciousness, Harvard University Press, Cambridge (MA).

11. Koch, C. (2004) The Quest for Consciousness: a Neurobiological Approach, Roberts and Company Publishers, Englewood.

12. Barbour, J. (1999) The End of Time: the Next Revolution in Physics, Oxford University Press, Oxford.

13. Healey, R. (2002) Can physics coherently deny the reality of time, in C. Callender (ed.), Time, Reality and Experience, Cambridge University Press, Cambridge, pp. 293-316.

14. Sri Nisargadatta Maharaj (1973) I Am That, Acorn Press, Durham, p. 39. 